\title{
Power Network Analysis Using an Adaptive Algebraic Multigrid Approach
}

\author{
Zhengyong Zhu, Bo Yao, Chung-Kuan Cheng \\ CSE Department, University of California, San Diego \\ 9500 Gilman Dr. \\ La Jolla, CA 92093-0114 \\ \{zzhu,byao,kuan\}@cs.ucsd.edu
}

\begin{abstract}
In this paper, we introduce an efficient analysis method for the power network of general topology. The new approach is based on algebraic multigrid (AMG) method that can avoid the slow convergence of basic iterative methods. An innovative adaptive coarsening scheme is employed to further speed up the performance, taking advantage of the spatial variation of power supply noise. Experimental results show that our method is more than 100 times faster than SPICE3.
\end{abstract}

\section{Categories and Subject Descriptors \\ B.7.2 [Design Aids]: Simulation}

\section{General Terms}

Algorithms, Performance, Experimentation

\section{Keywords}

Algebraic multigrid method, Adaptive Analysis, Power distribution network, Circuit Simulation

\section{INTRODUCTION}

Power network analysis covers voltage drop, voltage oscillation, and electromigration [1,2]. Excessive voltage drops reduce the switching speed as well as the noise margins of circuits and cause logic failures. Electromigration can decrease the chip lifetime [3]. Previous on-chip power grid analysis focuses on IR-drop caused by the resistance of power network. With the signal frequency increasing rapidly, the $j \omega L$ of on-chip power network becomes comparable with the resistance. Hence the inductance effect cannot be ignored. Moreover voltage oscillation may occur when power network resonance frequency drops to the range of the signal frequency [5].

One bottleneck of power network analysis is the tremendous amount of elements in power network. Direct methods such as LU decomposition used in SPICE are thus prohibitive. To address this bottleneck, M. Zhao and Y. Cao used hierarchical macro modeling or model order reduction techniques in [6][7]; H. Chen divided the chip into $100 * 100$ equipotential segments to reduce the complexity in [8]; Y. Lee adopted the ADI concepts in [9].

Permission to make digital or hard copies of all or part of this work for personal or classroom use is granted without fee provided that copies are not made or distributed for profit or commercial advantage and that copies bear this notice and the full citation on the first page. To copy otherwise, or republish, to post on servers or to redistribute to lists, requires prior specific permission and/or a fee.

DAC 2003, June 2-6, 2003, Anaheim, California, USA

Copyright 2003 ACM 1-58113-688-9/03/0006...\$5.00.
Some sparse matrix techniques have also been applied to the power network simulation. T. Chen employed a Preconditioned Conjugate Gradient (PCG) solver in $[10,18]$ and Nassif developed a multigrid method in [11][12].

In this paper, we present an adaptive approach for power grid analysis based on algebraic multigrid (AMG) method [15]. There are two kinds of multigrid methods: geometric multigrid and algebraic multigrid. Geometric multigrid requires regular mesh structure but has less coarse grid reduction cost while algebraic multigrid (AMG) can handle problems in general topology but requires heavier coarsening overhead [15].

The main differences between our approach and the multigrid method in [11][12] are

1) Our approach covers self and mutual inductances, while [11][12] can only handle RC networks. This is due to the limitation of geometric multigrid coarsening algorithm used in [11][12]. Since multigrid method requires the system matrix to be symmetric positive definite (S.P.D.) which circuits with inductors cannot satisfy using Modified Nodal Analysis method (MNA), reformulation as discussed in section 2 is needed. But the topology of reformulated system matrix is no longer the same as original circuit topology. It is not easy to derive coarse level grid directly from circuit topology in this kind of situation even if the power network is a regular mesh.

2) Our coarsening scheme makes no assumption about the topology of power network. While in [11][12], special consideration is needed for irregular power networks.

3) $[11][12]$ ignored the error smoothing operation and only executed one multigrid iteration cycle. Although it may not result in much error for well-designed power network, accuracy cannot always be guaranteed for general cases. We keep the smoothing operations and iterate the multigrid cycles until the norm of the residue is less than a threshold.

4) We adopt an adaptive coarsening scheme. The coarse grid is determined by the matrix structure and the circuit activities adaptively. Adaptive coarsening makes it possible to assign more computation to the active subnetworks of the power network than to the rest circuit.

\section{OVERVIEW}

We focus on power source network with the assumption that power and ground can be separated. Usually power network is an irregular mesh as shown in Figure 2.1. Every intersection node has a ground capacitance. Between neighboring intersection nodes is resistor or resistor and inductor in series. Mutual inductance can also be included. The devices with activities are modeled as timevarying current sources [8]. 
When only RC model is included, system equation can be formulated as

$$
C \dot{X}(t)+G X(t)=U(t),
$$

where $\mathrm{X}$ is the vector of nodal voltages.

Appling trapezoidal approximation with time step $\mathrm{h}$ to equation (2.1), we have

$$
\left(G+\frac{2}{h} C\right) X(t+h)=-\left(G-\frac{2}{h} C\right) X(t)+U(t)+U(t+h)
$$

The left hand side matrix in (2.2) is symmetric and positive definite which makes the iterative methods converge quickly.

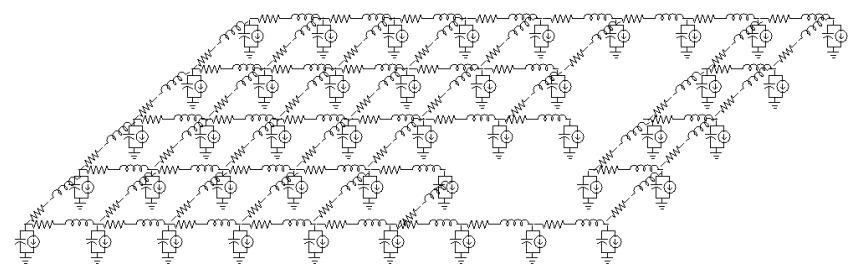

Figure 2.1 Power Network Structure

When inductance is included, the system matrix equation becomes:

$$
\hat{C} \dot{\hat{X}}(t)+\hat{G} \hat{X}(t)=\hat{U}(t)
$$

where $\hat{C}=\left[\begin{array}{ll}C & 0 \\ 0 & L\end{array}\right], \hat{X}=\left[\begin{array}{l}V \\ I\end{array}\right], \hat{U}=\left[\begin{array}{c}U \\ 0\end{array}\right]$ and $\hat{G}=\left[\begin{array}{cc}G & -A_{l}{ }^{T} \\ A_{l} & 0\end{array}\right]$

Equation (2.3) can be rewritten as:

$$
\left[\begin{array}{cc}
C & 0 \\
0 & L
\end{array}\right]\left[\begin{array}{c}
\dot{V} \\
\dot{I}
\end{array}\right]+\left[\begin{array}{cc}
G & -A_{l}^{T} \\
A_{l} & 0
\end{array}\right]\left[\begin{array}{l}
V \\
I
\end{array}\right]=\left[\begin{array}{c}
U \\
0
\end{array}\right]
$$

Appling trapezoidal approximation with time step $\mathrm{h}$, the solution of equation (2.4) is derived by

$\left[\begin{array}{cc}\frac{2 C}{h}+G & -A_{l}^{T} \\ A_{l} & \frac{2 L}{h}\end{array}\right]\left[\begin{array}{l}V(t+h) \\ I(t+h)\end{array}\right]=\left[\begin{array}{cc}\frac{2 C}{h}-G & A_{l}^{T} \\ -A_{l} & \frac{2 L}{h}\end{array}\right]\left[\begin{array}{c}V t) \\ I(t)\end{array}\right]+\left[\begin{array}{c}U(t+h)+U(t) \\ 0\end{array}\right]$

Although MNA method can handle elements without admittance description, the transient analysis system matrix in (2.5) is no longer symmetric and positive definite when the inductance is included because of the introduction of current variables.

Since Multigrid as well as PCG method requires the matrix to be symmetric positive definite, some extra processing is needed to reformulate the system [16]. Similar to the method used in [10], we split the variable vector into nodal voltage vector and branch current vector. Using block matrix operations, we decompose (2.5) into two iteration formulas (2.6) for nodal voltages and branch currents, respectively.

$$
\Rightarrow\left\{\begin{array}{c}
\left(\frac{2 C}{h}+G-\frac{h}{2} A_{l}^{T} L^{-1} A_{l}\right) V(t+h)= \\
\left(\frac{2 C}{h}-G+\frac{h}{2} A_{l}^{T} L^{-1} A_{l}\right) V(t)+[U(t)+U(t+h)]+2 A_{l}^{T} I(T) \\
I(t+h)=I(t)-\frac{h}{2} L^{-1} A_{l}[V(t+h)+V(t)]
\end{array}\right.
$$

In equation (2.6), $L^{-1}$ corresponds to the $\mathrm{K}$ matrix [17], where the matrix inversion overhead is reduced by sparsification methods.

If the inductance matrix is symmetric and positive definite (S.P.D.), we can prove that the system matrix is still S.P.D. This also holds for forward Euler, backward Euler integration approximation methods. Note that the topology of reformulated system matrix in (2.6) is no longer the same as original circuit topology. Geometric based coarse grid reduction algorithm cannot be applied to RLC network directly.

\section{Multigrid Method}

The multigrid method is an efficient technique first widely used for solving partial differential equations [15]. The basic idea of multigrid method is to map the hard-to-damp low frequency error at fine level to easy-to-damp high frequency error at coarse level, solve the mapped problem at coarse level, and then map the error correction of coarse level back to fine level. The mapping operator from fine to coarse level is called restriction operator $I_{h}^{2 h}$, and the mapping operator from coarse to fine level is called interpolation operator $I_{2 h}^{h}$. Here the subscription represents the level. At each level, high frequency error is erased by a smoothing operator that is a forward iterative method such as Gauss-Seidel. The construction of hierarchical grid structure stops at the level when the reduced matrix can be quickly solved by LU decomposition. Once we find the exact solution of the problem at the coarsest level, we perform the interpolation from the coarsest level back to the find grid. An iteration of restriction from the fine grid to the coarsest grid and interpolation from the coarsest grid back to the fine grid is called a single multigrid cycle, which will iterate several times till converge.

\subsection{Geometric Multigrid vs. Algebraic Multigrid}

There are two kinds of multigrid methods: geometric multigrid and algebraic multigrid (AMG). Geometric multigrid method requires regular mesh structure. Its coarse grid reduction algorithm is defined geometrically, e.g. coarse node is selected every other fine node.

If we want to handle problems with irregular structure, AMG is a good alternative of geometric multigrid method. The coarsening and interpolation operation of AMG are based on matrix itself. This overhead makes AMG less efficient than geometric multigrid if the problem analyzed has regular mesh structure.

In geometric multigrid, the smoothing operation at each level is important to the convergence. The error after smoothing should be geometrically smooth, in other words, the local error among adjacent nodes should be small. While in AMG, the interpolation plays the crucial role. The convergence rate actually depends on the coarse grid construction and inter-level mapping operators [14].

\subsection{AMG Interpolation \& Restriction Operator}

Since AMG has no grid concept in mind, the inter-level mapping operators have to be determined based on the matrix. In AMG, smooth error means error components with relatively small residuals (3.1) [15], which means after several iterations of smoothing operation, the residue is small but the error decreases very slowly.

$$
A e \approx 0
$$

Equation (3.1) can be rewritten as (3.2). Hence the error of fine node can be well represented by linear combinations of its neighbors' error.

$$
a_{i i} e_{i} \approx-\sum_{j \neq i} a_{i j} e_{j}
$$


If coarse and fine nodes have already been defined, we can further approximate the error of fine node by errors of only its coarse node neighbors. Although there are many other complicated interpolation methods, we find that this simple approach works well for the power grid problem. From (3.2), we can construct the interpolation operator $I_{2 h}^{h}$. Because of the symmetry of the mapping between levels in two directions, the restriction operator $I_{h}^{2 h}$ is the transposition of the interpolation operator, i.e. $I_{2 h}^{h^{T}}=I_{h}^{2 h}$. The coarse level matrix can be computed as $A^{2 h}=I_{2 h}^{h} A^{h} I_{h}^{2 h}$. Consequently, coarse level matrix $A^{2 h}$ remains symmetric positive definite.

If the matrix is symmetric and positive definite, convergence of AMG can be guaranteed as long as the smoothing operation converges at each level. A detailed proof can be found in [13].

\section{ADAPTIVE COARSENING SCHEME}

\subsection{Spatial Variation of Power Supply Noise}

For typical power grid, the RLC values are quite uniform at the same layer. But due to the non-uniform power densities and the timing of the switching events, power supply noise exhibits spatial variation, which means some nodes of the power network have more rapid nodal voltage changes, or in other words, these nodes are more active than the rest circuit.

Adaptive concepts can be integrated with the coarsening scheme in multigrid. Some adaptive methods like FAC [19] and MLAT [20] can be applied to geometric multigrid method. The basic idea behind these adaptive methods is that active regions should have finer grid structure, since active subnetworks need more computation to model their behavior accurately.

In our adaptive coarsening scheme, active regions have relatively finer grid at coarse level. The coarse grid nodes are determined adaptively by the circuit activities as well as by the matrix. The coarse grid consists of two kinds of nodes: non-adaptive and adaptive coarse nodes. Non-adaptive coarse nodes are selected by coloring scheme [15] according to the matrix. Adaptive coarse nodes are determined by the circuit activities.

\subsection{Coloring Scheme for Non-Adaptive Coarse Nodes}

A two-level coloring scheme is explained below for sake of clarity. In practice, the coloring scheme is executed at every level except the coarsest one.

Initially, the potential of each node is set to its degree. The node with the maximum potential is selected to be a coarse node and all its unassigned neighboring nodes are set as fine nodes. For each newly set fine node, we increase the potential of its neighbor by 1 . The process is repeated until every node has been assigned as fine or coarse node. At the end, each fine node has at least one neighboring coarse node.

\subsection{Adaptive Coarse Nodes Selection}

Adaptive coarse nodes are selected according to the activities of circuits. One good candidate for the measurement of the impact of circuit activities is the first order derivative of nodal voltages that can be approximated from equation (2.4) for RLC network.

For RLC networks as shown in Fig 2.1, since not every node has a ground capacitor, we split voltage vector $\mathrm{V}$ into two separate voltage vectors $V_{1}$ and $V_{2}$, where $V_{1}$ is the set of nodes with ground capacitor and $\mathrm{V}_{2}$ is the set of nodes between resistance and inductance in branches. We then calculate the first order derivative of nodal voltage in $\mathrm{V}_{1}$.

Rewriting equation (2.4), we have

$$
\left[\begin{array}{ccc}
C^{\prime} & & \\
& 0 & \\
& & L
\end{array}\right]\left[\begin{array}{c}
\dot{V}_{1} \\
\dot{V}_{2} \\
\dot{I}
\end{array}\right]=-\left[\begin{array}{ccc}
G_{11} & G_{12} & -A_{l 1}{ }^{T} \\
G_{21} & G_{22} & -A_{l 2}{ }^{T} \\
A_{l 1} & A_{l 2} & 0
\end{array}\right]\left[\begin{array}{c}
V_{1} \\
V_{2} \\
I
\end{array}\right]+\left[\begin{array}{c}
U \\
0 \\
0
\end{array}\right]
$$

The first order derivative of intersection node voltage can be approximated as

$$
\dot{V}_{1}(t+h)=-C^{\prime-1}\left[G_{11} V_{1}(t)+G_{12} V_{2}(t)-A_{l 1}^{T} I(t)+U(t+h)\right]
$$

The inversion of capacitance matrix is easy because the capacitance matrix is actually a diagonal matrix.

Those nodes with relatively larger voltage derivative are selected as adaptive coarse nodes. We apply adaptive coarsening at the finest level. The coarse grid selection at other levels is determined solely by the coloring scheme, because active components with finer first level coarse grid will still have relatively finer grid on the next coarse levels. Figure 4.1 shows the comparison of nonadaptive and adaptive coarsening structure. Darker color means coarser level. The circuit activity focuses on the up-right corner of the circuits. Active region has finer grid than the other region in every grid level.
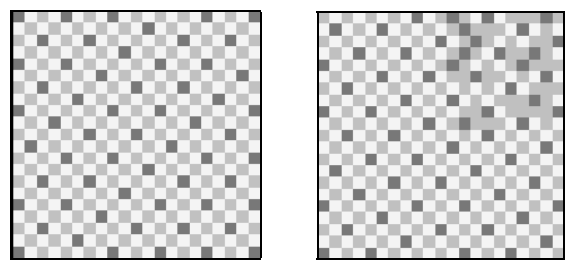

Figure 4.1 Non-Adaptive (left) and Adaptive (right) Coarsening Structure

\section{EXPERIMENTAL RESULTS}

The proposed approach is implemented in ANSI C. The experiments are executed on a SUN Blade100 $(300 \mathrm{MHz})$ workstation with $2 \mathrm{~GB}$ memory.

We set the number of pre-smoothing and post-smoothing iteration to 3 at each level and the multigrid iteration termination control residue norm to $1 \times 10^{-10}$. Gauss-Seidel method acts as smoothing operator at each level.

Table 1 shows the DC analysis runtime of SPICE3 and our approach, which is more than 100 times faster than SPICE3 for large circuits. Table 2 compares the transient analysis runtime of SPICE3, non-adaptive AMG, and adaptive AMG. The timevarying currents are modeled as triangular waveforms with $2 \mathrm{~mA}$ peak current and 40ps rising and falling time [21]. Current sources are not evenly distributed and the timings are also different. We execute the transient analysis for 5ns. Experimental results show that adaptive AMG is faster than non-Adaptive AMG. The speedup against the non-adaptive AMG is not very obvious because we only take advantage the faster convergence rate of adaptive grid structure now. More improvement is expected after we extend the adaptive concept to smoothing operations at each level. Our approach runs about 20 times faster than SPICE3 for transient analysis. The performance speedup is comparable with the PCG approach in [10]. The number of multigrid iterations 
dose not increase rapidly with the problem size. Actually the number of iterations is independent of the problem size, which cannot be proved but is observed in many cases.

Table1 DC Analysis Runtime Comparison (sec)

\begin{tabular}{|c|c|c|c|}
\hline Nodes & SPICE & AMG & Speedup \\
\hline 1706 & 1.68 & 0.17 & 9.8 \\
\hline 2637 & 3.91 & 0.28 & 14 \\
\hline 5105 & 15.01 & 0.57 & 26.3 \\
\hline 10322 & 54.44 & 0.98 & 55.5 \\
\hline 40842 & 708.22 & 3.93 & 180.2 \\
\hline 91562 & $\mathrm{X}$ & 8.98 & \\
\hline
\end{tabular}

Table 2. Transient Analysis Runtime Comparison (sec)

\begin{tabular}{|c|c|c|c|c|}
\hline Nodes & SPICE & AMG & Adaptive AMG & Adaptive Speedup \\
\hline 1706 & 18.06 & 1.36 & 1.36 & 13.2 \\
\hline 2637 & 41.23 & 4.08 & 3.78 & 10.9 \\
\hline 5105 & 122.1 & 9.13 & 8.89 & 13.7 \\
\hline 10322 & 456.42 & 18.7 & 18.1 & 25.2 \\
\hline 40842 & 5048.5 & 165.1 & 155.2 & 32.5 \\
\hline
\end{tabular}

Figure 5.1 compares the transient analysis voltage waveforms of one node from SPICE and our adaptive AMG approach. The waveforms are almost the same.
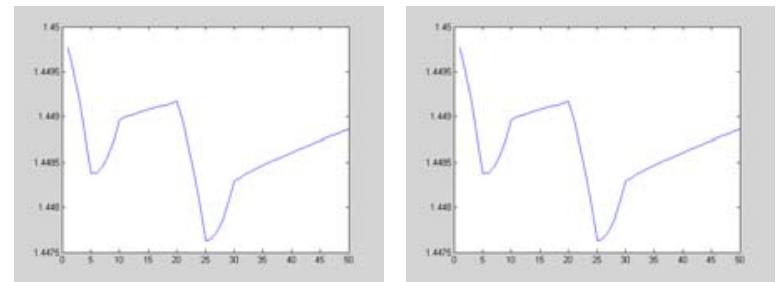

Fig 5.1 Comparison of voltage waveforms from SPICE (left) and Adaptive AMG (right)

\section{CONCLUSION AND FUTURE WORK}

In this paper, we present an adaptive AMG approach for power network analysis. The spatial variation of power supply noise is utilized to further speed up the performance. The new approach can handle power network of general topology with self and mutual inductances. Experimental result is very promising. In the future work, we will extend the adaptive concept to the smoothing operation at each grid level to fully explore the potential of adaptive multigrid.

\section{ACKNOWLEDGEMENTS}

The authors gratefully acknowledge the funding provided by NSF MIP-9987678 and California State MICRO Grant. They would like to thank Prof. E.S. Kuh for his valuable advice.

\section{REFERENCES}

[1] S. Lin, N. Chang, "Challenges in Power-Ground Integrity", IEEE/ACM International Conference on Computer Aided Design. 2001.

[2] S. Bobba, T. Thorp, K. Aingaran, D. Liu, "IC power distribution challenges," IEEE/ACM International Conference on Computer Aided Design 2001

[3] J. R. Balck, "Electromigration Failure Modes in Aluminum Metalization for Semiconductor Devices", Proc. IEEE, pp. 1587-1594, Sep. 1969
[4] G. A. Katopis, "Delta-I Noise Specification for a Highperformance Computing Machine," Proc. of the IEEE, vol. 73, pp.1405-1415, 1985

[5] S. Taylor, "The Challenge of Designing Global Signals in UDSM CMOS," IEEE Custom Integrated Circuits Conference, San Diego, CA 1999

[6] M. Zhao, R. V. Panda, S. S. Sapatnekar, D. Blaauw, "Hierarchical analysis of power distribution networks," IEEE Transactions on Computer-Aided Design of Integrated Circuits and Systems, vol.21, (no.2), IEEE, Feb. 2002. p.159-68.

[7] Y. Cao, Y. Lee, T. Chen, and C. Chen, "HiPRIME: Hierarchical and Passivity Reserved Interconnect Macromodeling Engine for RLKC Power Delivery", IEEE/ACM Design Automation Conference 2002

[8] H. Chen, J. Neely, "Interconnect and Circuit Modeling Techniques for Full-Chip Power Supply Noise Analysis", IEEE Transactions on Components, Packaging and Manufacturing Technology, Part B, Vol21, No.3, August 1998

[9] Y. Lee and C. Chen, "Power Grid Transient Simulation in Linear Time Based on Transmission-Line-Modeling Alternating-Direction-Implicit Method," IEEE/ACM International Conference on Computer Aided Design, 2001

[10] T. Chen and C. Chen, "Efficient Large-Scale Power Grid Analysis Based on Preconditioned Krylov-Subspace Iterative Methods", IEEE/ACM Design Automation Conference, 2001.

[11] S. R. Nassif et al, "Fast Power Grid Simulation", IEEE/ACM Design Automation Conference 2000.

[12] J. N. Kozhaya, S. R. Nassif, F. N. Najm, "Multigrid-like Technique for Power Grid Analysis", IEEE/ACM International Conference on Computer Aided Design. 2001.

[13]K. Stuben, "Algebraic Multigrid: An Introduction with Applications", GMD Report No.53, March 1999

[14] K. Stuben, "A review of algebraic multigrid" Journal of Computational and Applied Mathematics, vol.128, (no.1-2), Elsevier, 1 March 2001. p.281-309

[15] W. L. Briggs, “A Multigrid Tutorial”, SIAM 2000

[16] "Design of high-performance microprocessor circuits," IEEE Press, 2001

[17] A. Devgan, .et al, "How to Efficiently Capture On-Chip Inductance Effects: Introducing a New Circuit Element K", IEEE/ACM International Conference on Computer Aided, Nov 2000, pp 150-155

[18] G. H. Golub, "Matrix Computations", Johns Hopkins University Press, 1996

[19] S. F. McCormick,, "Multilevel Adaptive Methods for Partial Differential Equations," vol.6, Frontiers in Applied Mathematics, SIAM, Philadelphia, 1989

[20] A. Brandt, "Multi-level adaptive solutions to boundary value problems," Math. Comput., 31, 1977, pp.333-390

[21] S. Zhao, K. Roy, C. K. Koh "Frequency domain analysis of switching noise on power supply network," IEEE/ACM International Conference on Computer Aided Design. 2000. 